PROCEEDINGS OF THE

AMERICAN MATHEMATICAL SOCIETY

Volume 137, Number 4, April 2009, Pages 1275-1283

S 0002-9939(08)09622-6

Article electronically published on October 9, 2008

\title{
AN ALGEBRAIC INDEPENDENCE RESULT FOR EULER PRODUCTS OF FINITE DEGREE
}

\author{
ALEXANDRU ZAHARESCU AND MOHAMMAD ZAKI
}

(Communicated by Ken Ono)

\begin{abstract}
We investigate the algebraic independence of some derivatives of certain multiplicative arithmetical functions over the field $\mathbb{C}$ of complex numbers.
\end{abstract}

\section{INTRODUCTION}

In this paper we consider arithmetical functions defined over the field of complex numbers, and their associated Dirichlet series. Let $r \geq 1$ be an integer and write $A_{r}=A_{r}(\mathbb{C})=\left\{f: \mathbb{N}^{r} \rightarrow \mathbb{C}\right\}$. Given $f, g \in A_{r}$, define the convolution $f * g$ of $f$ and $g$ by

$$
(f * g)\left(n_{1}, \ldots, n_{r}\right)=\sum_{d_{1} \mid n_{1}} \ldots \sum_{d_{r} \mid n_{r}} f\left(d_{1}, \ldots, d_{r}\right) g\left(\frac{n_{1}}{d_{1}}, \ldots, \frac{n_{r}}{d_{r}}\right) .
$$

Then $\mathbb{C}$ has a natural embedding in the ring $A_{r}$, and $A_{r}$ with addition and convolution defined as above becomes a $\mathbb{C}$-algebra. The ring $A_{1}$ has been studied from various points of view by a number of authors. We mention in this connection the work of Cashwell and Everett [4, who proved that $\left(A_{1},+,.\right)$ is a unique factorization domain. Schwab and Silberberg 12 constructed an extension of $\left(A_{1},+,.\right)$ which is a discrete valuation ring. Alkan and the authors [1 generalized this construction and provided a family of extensions of $A_{r}$ which are discrete valuation rings. For other work on rings of arithmetical functions the reader is referred to [5, 6, 9], 12, [13, 10, 11, 2]. In [1], it was shown that for any completely additive arithmetical function $\psi \in A_{r}$, the map $D_{\psi}: A_{r} \rightarrow A_{r}$ defined by $D_{\psi}(f)\left(n_{1}, \ldots, n_{r}\right)=f\left(n_{1}, \ldots, n_{r}\right) \psi\left(n_{1}, \ldots, n_{r}\right)$, for all $n_{1}, \ldots, n_{r} \in \mathbb{N}$, is a derivation on $A_{r}$. It was also proved in 1 that for any multiplicative function $f \in A_{r}$, any completely additive function $\psi \in A_{r}$, and any $n_{1}, \ldots, n_{r} \in \mathbb{N}$ not all prime powers, $\frac{D_{\psi}(f)}{f}\left(n_{1}, \ldots, n_{r}\right)=0$, where $\frac{D_{\psi}(f)}{f}$ is viewed as $D_{\psi}(f) * f^{-1}$. In this connection, a natural line of investigation would be to study the action of $D_{\psi}$ on the subring $\mathbb{C}[f]$ of $A_{r}$ generated over $\mathbb{C}$ by a given multiplicative function $f \in A_{r}$, for any $\psi$ as above. From this point of view, the first issue that arises is to consider the image of $\mathbb{C}[f]$ through $D_{\psi}$, and identify the intersection of $D_{\psi}(\mathbb{C}[f])$ and $\mathbb{C}[f]$. We will do this for a special class of multiplicative functions $f$ which are of particular interest, namely, those which have Euler factors of finite degree.

Received by the editors January 25, 2008, and, in revised form, May 1, 2008.

2000 Mathematics Subject Classification. Primary 11J85, 13J99.

Key words and phrases. Arithmetical functions, algebraic independence.

(C)2008 American Mathematical Society 1275

Reverts to public domain 28 years from publication 
Fix $\psi \in A_{r}$. Assume that $\psi$ is completely additive and satisfies

$$
\left|\psi\left(n_{1}, \ldots, n_{r}\right)\right| \rightarrow \infty,
$$

as $n_{1}+\cdots+n_{r} \rightarrow \infty$. For any $g \in A_{r}$, any prime number $p$, and any integer $k \in\{1, \ldots, r\}$, let $g_{p, k, r} \in A_{1}$ be the function defined as follows. Let $m \in \mathbb{N}$. If $m$ is not a power of the prime $p$, then $g_{p, k, r}(m)=0$. If $m=p^{n}$ for some nonnegative integer $n$, let

$$
g_{p, k, r}\left(p^{n}\right)=g\left(1, \ldots, 1, p^{n}, 1, \ldots, 1\right),
$$

where $p^{n}$ occurs at the $k$-th component of the tuple $\left(1, \ldots, 1, p^{n}, 1, \ldots, 1\right)$ on the rightside of (1.2). Given a multiplicative function $f \in A_{r}$, we say that $f$ has an Euler factor of finite degree at a prime number $p$ provided there exists $k \in\{1, \ldots, r\}$ and $m \in \mathbb{N}$ and nonzero complex numbers $a_{1}, \ldots, a_{m}$ such that the Dirichlet series associated to the arithmetical function $f_{p, k, r}$ is given by

$$
\sum_{n=1}^{\infty} \frac{f_{p, k, r}(n)}{n^{s}}=\frac{1}{\left(1-\frac{a_{1}}{p^{s}}\right) \cdots\left(1-\frac{a_{m}}{p^{s}}\right)} .
$$

As a matter of terminology, we will call the above Euler factor trivial if $m=0$ and respectively nontrivial if $m \geq 1$. We will prove the following result.

Theorem 1. Let $\psi \in A_{r}$ be completely additive and satisfy

$$
\left|\psi\left(n_{1}, \ldots, n_{r}\right)\right| \rightarrow \infty,
$$

as $n_{1}+\cdots+n_{r} \rightarrow \infty$. Let $f \in A_{r}$ be multiplicative and such that for infinitely many prime numbers $p, f$ has an Euler product of finite degree at $p$ as defined above. Then for any distinct nonnegative integers $i$, and $j$, the derivations $D_{\psi}^{i}(f)$ and $D_{\psi}^{j}(f)$ of $f$ of orders $i$ and $j$ respectively are algebraically independent over $\mathbb{C}$.

As a consequence of this result, for $\psi$ and $f$ as above, the arithmetical function which is constant and equal to zero is the only common element of $D_{\psi}(\mathbb{C}[f])$ and $\mathbb{C}[f]$.

Corollary 1. Let $\psi$ and $f$ be elements of $A_{r}$ satisfying the assumptions in Theorem 1 . Let $\mathbb{C}[f]$ be the subring of $A_{r}$ generated over $\mathbb{C}$ by $f$. Then,

$$
D_{\psi}(\mathbb{C}[f]) \cap \mathbb{C}[f]=0 .
$$

We end this section with some examples. Let $r=1$, and let $\psi_{0} \in A_{1}$ be the completely additive function given by $\psi_{0}(n)=-\log n$ for all $n \in \mathbb{N}$. Then condition (1.3) is satisfied. Next, let $f=\chi$ be a Dirichlet character. So $f$ satisfies the condition in Theorem 1 with $m=1$, for all but finitely many primes (where the corresponding Euler factor is trivial). Then Theorem 1 applies, and it shows that the derivations $D_{\psi_{0}}^{(i)}(\chi)$ and $D_{\psi_{0}}^{(j)}(\chi)$ of $\chi$ of orders $i$ and $j$ are algebraically independent for any nonnegative distinct integers $i$ and $j$. Moreover, by the standard isomorphism which sends any arithmetical function $h \in A_{1}(\mathbb{C})$ to its associated Dirichlet series $H(s)=L(s, h)=\sum_{1}^{\infty} \frac{h(n)}{n^{s}}$, and also sends $D_{\psi_{0}}(h)$ to $\frac{d}{d s}(H(s))$, we see that for any nonnegative distinct integers $i$ and $j$, the functions $L^{(i)}(s, \chi)$ and $L^{(j)}(s, \chi)$ are algebraically independent over $\mathbb{C}$. 
For another example, let us again take $r=1, f=\chi$, and $\psi_{0}$ as above. Also fix a prime $p$ such that $\chi(p) \neq 0$. Next, let $\chi_{p} \in A_{1}(\mathbb{C})$ be defined by

$$
\chi_{p}(n)= \begin{cases}\chi(n), & \text { if } n=p^{m}, m \geq 1 \\ 0, & \text { otherwise }\end{cases}
$$

Note that

$$
\begin{aligned}
D_{\psi_{0}}\left(\chi_{p}\right)\left(p^{m}\right) & =(-m \log p)\left(\chi_{p}(p)\right)^{m} \\
& =(-\log p)\left(\chi_{p}(p)\right)^{m}\left(\left(\sum_{d \mid p^{m}} 1\right)-1\right) \\
& =(\log p)\left(\chi_{p}^{2}\left(p^{m}\right)-\chi_{p}\left(p^{m}\right)\right) .
\end{aligned}
$$

One finds that

$$
D_{\psi_{0}}\left(\mathbb{C}\left[\chi_{p}\right]\right)=\left(\chi_{p}^{2}-\chi_{p}\right) \mathbb{C}\left[\chi_{p}\right]
$$

Thus Corollary 1, and therefore also Theorem 1 fails in this case. But $\chi_{p}$ does not satisfy the hypothesis of Theorem 1 either.

Other interesting examples arise from the theory of modular forms. For a nice treatment of this subject the reader is referred to the recent monograpgh of Ono [7. Let $f(z)$ be a newform (or normalized Hecke eigenform) of weight $k$ in $S_{k}\left(\Gamma_{1}(N), \chi\right)$ which has Fourier expansion

$$
f(z)=\sum_{n=1}^{\infty} a_{f}(n) e^{2 \pi i n z}, \operatorname{Im} z>0 .
$$

The Fourier coefficients $a_{f}(n)$ form a multiplicative arithmetical function. The associated $L$-function is given by

$$
L(s, f)=\sum_{n=1}^{\infty} a_{f}(n) n^{-s}
$$

where $s \in \mathbb{C}$ is a complex variable. Here $L(s, f)$ has an Euler product expansion

$$
L(s, f)=\prod_{p}\left(1-a_{f}(p) p^{-s}+\chi(p) p^{k-1-2 s}\right)^{-1}=\prod_{p} \frac{1}{\left(1-\frac{\alpha_{p} p^{\frac{k-1}{2}}}{p^{s}}\right)\left(1-\frac{\beta_{p} p^{\frac{k-1}{2}}}{p^{s}}\right)},
$$

where the product is taken over all primes, $\alpha_{p}+\beta_{p}=a_{f}(p) p^{\frac{1-k}{2}}$, and $\alpha_{p} \beta_{p}=\chi(p)$.

For example, one can take the Ramanujan tau function $\tau(n)$, defined in terms of the Delta function

$$
\Delta(z)=\sum_{n=1}^{\infty} \tau(n) q^{n}=q \prod_{n=1}^{\infty}\left(1-q^{n}\right)^{24}, \quad q=e^{2 \pi i z}
$$

which is the unique normalized cusp form of weight 12 on $S L_{2}(\mathbb{Z})$. The Euler product expansion of the $L$-series associated to $\Delta(z)$ is given by

$$
L(s, \Delta)=\prod_{p}\left(1-\tau(p) p^{-s}+p^{11-2 s}\right)^{-1}=\prod_{p} \frac{1}{\left(1-\frac{\alpha_{p} p^{\frac{11}{2}}}{p^{s}}\right)\left(1-\frac{\beta_{p} p^{\frac{11}{2}}}{p^{s}}\right)},
$$

where the product is taken over all primes, $\alpha_{p}+\beta_{p}=\tau(p) p^{-\frac{11}{2}}$, and $\alpha_{p} \beta_{p}=1$.

The conditions in Theorem 1 are satisfied in this case, and therefore any two derivatives of $L(s, f)$ are algebraically independent over $\mathbb{C}$. 
Theorem 1 applies, more generally, to the case when $f$ is an automorphic cusp form on $G L_{m} / \mathbb{Q}, m \geq 1$. Its $L$-function $L(s, f)$ has an Euler product of degree $m$ : $L(s, f)=\prod_{p} L\left(s, f_{p}\right)$, where

$$
L\left(s, f_{p}\right)=\frac{1}{\prod_{j=1}^{m}\left(1-\frac{\alpha_{j, f}(p)}{p^{s}}\right)} .
$$

By Theorem 1, any two derivatives of $L(s, f)$ are algebraically independent over $\mathbb{C}$.

\section{Preliminaries}

Let $r$ be a positive integer and denote as above $A_{r}=\left\{f: \mathbb{N}^{r} \rightarrow \mathbb{C}\right\}$. We say that an arithmetical function $f \in A_{r}$ is multiplicative provided one has

$$
f\left(n_{1} m_{1}, \ldots, n_{r} m_{r}\right)=f\left(n_{1}, \ldots, n_{r}\right) f\left(m_{1}, \ldots, m_{r}\right),
$$

for any $n_{1}, \ldots, n_{r}, m_{1}, \ldots, m_{r} \in \mathbb{N}$ satisfying $\left(n_{1}, m_{1}\right)=\cdots=\left(n_{r}, m_{r}\right)=1$. We say that $f \in A_{r}$ is completely multiplicative provided

$$
f\left(n_{1} m_{1}, \ldots, n_{r} m_{r}\right)=f\left(n_{1}, \ldots, n_{r}\right) f\left(m_{1}, \ldots, m_{r}\right),
$$

for any $n_{1}, \ldots, n_{r}, m_{1}, \ldots, m_{r} \in \mathbb{N}$. Similarly we say that a function $f \in A_{r}(R)$ is additive provided

$$
f\left(n_{1} m_{1}, \ldots, n_{r} m_{r}\right)=f\left(n_{1}, \ldots, n_{r}\right)+f\left(m_{1}, \ldots, m_{r}\right),
$$

for any $n_{1}, \ldots, n_{r}, m_{1}, \ldots, m_{r} \in \mathbb{N}$ satisfying $\left(n_{1}, m_{1}\right)=\cdots=\left(n_{r}, m_{r}\right)=1$. We call a function $f \in A_{r}$ completely additive provided

$$
f\left(n_{1} m_{1}, \ldots, n_{r} m_{r}\right)=f\left(n_{1}, \ldots, n_{r}\right)+f\left(m_{1}, \ldots, m_{r}\right),
$$

for any $n_{1}, \ldots, n_{r}, m_{1}, \ldots, m_{r} \in \mathbb{N}$. For any completely additive function $\psi \in A_{r}$, the map $D_{\psi}: A_{r} \rightarrow A_{r}$ defined by

$$
D_{\psi}(f)\left(n_{1}, \ldots, n_{r}\right)=f\left(n_{1}, \ldots, n_{r}\right) \psi\left(n_{1}, \ldots, n_{r}\right),
$$

for all $n_{1}, \ldots, n_{r} \in \mathbb{N}$, satisfies the following properties (see [1]). For all $f, g \in A_{r}$ and $c \in \mathbb{C}$,

(a) $D_{\psi}(f+g)=D_{\psi}(f)+D_{\psi}(g)$,

(b) $D_{\psi}(f g)=f D_{\psi}(g)+g D_{\psi}(f)$,

(c) $D_{\psi}(c f)=c D_{\psi}(f)$.

Consequently, $D_{\psi}$ is a derivation on $A_{r}$ over $\mathbb{C}$.

Every $f \in A_{r}$ has an associated formal Dirichlet series

$$
\bar{f}\left(s_{1}, \ldots, s_{r}\right)=\sum_{n_{1}, \ldots, n_{r} \in \mathbb{N}} \frac{f\left(n_{1}, \ldots, n_{r}\right)}{n_{1}^{s_{1}} \cdots n_{r}^{s_{r}}} .
$$

Let $\bar{A}_{r}$ be the ring of all such series with the usual addition and multiplication of series. The map $f \rightarrow \bar{f}$ is a ring isomorphism.

For any $g \in A_{r}$, a prime number $p$, and an integer $k \in\{1, \ldots, r\}$, let us denote by $\phi_{p, k, r}$ the map from $A_{r}$ into $A_{1}$ which sends $g$ to $g_{p, k}=g_{p, k, r} \in A_{1}$, where $g_{p, k, r}$ is defined as in Section 1. The mapping $\phi_{p, k, r}$ is a homomorphism of $\mathbb{C}$-algebras: for any $c \in \mathbb{C}$ and $g, h \in A_{r},(c g)_{p, k, r}=c g_{p, k, r},(g+h)_{p, k, r}=g_{p, k, r}+h_{p, k, r}$, and $(g * h)_{p, k, r}=g_{p, k, r} * h_{p, k, r}$. To see this, let $n \in \mathbb{N}$ and consider the $r$-tuple 
$\left(1, \ldots, 1, p^{n}, 1, \ldots, 1\right) \in \mathbb{N}^{r}$, where $p^{n}$ occurs at the $k$-th component of the tuple. Then,

$$
\begin{aligned}
(g * h)_{p, k, r}\left(p^{n}\right) & =(g * h)\left(1, \ldots, 1, p^{n}, 1, \ldots, 1\right) \\
& =\sum_{d \mid p^{n}} g(1, \ldots, d, 1, \ldots, 1) h\left(1, \ldots, \frac{p^{n}}{d}, 1, \ldots, 1\right) \\
& =\sum_{d \mid p^{n}} g_{p, k, r}(d) h_{p, k, r}\left(\frac{p^{n}}{d}\right) \\
& =g_{p, k, r} * h_{p, k, r}\left(p^{n}\right) .
\end{aligned}
$$

On the other hand, if $n$ is not a power of the prime $p$, then we have that

$$
(g * h)_{p, k, r}(n)=0=g_{p, k, r} * h_{p, k, r}(n) .
$$

Therefore, $(g * h)_{p, k, r}=g_{p, k, r} * h_{p, k, r}$. Similarly, one sees that $(c g)_{p, k, r}=c g_{p, k, r}$ and $(g+h)_{p, k, r}=g_{p, k, r}+h_{p, k, r}$.

Note that the homomorphism sending any $g \in A_{r}$ to $g_{p, k, r} \in A_{1}$ induces a homomorphism of $\bar{A}_{r}$ onto $\bar{A}_{1}$ which sends $\bar{g}\left(s_{1}, \ldots, s_{r}\right)$ to $\bar{g}_{p, k, r}(s)$. As an example, for $r=1$, this map sends the Riemann zeta function

$$
\zeta(s)=\sum_{n=1}^{\infty} \frac{1}{n^{s}}=\prod_{q} \frac{1}{1-\frac{1}{q^{s}}}
$$

to the function $\zeta_{p}(s)=\sum_{k=0}^{\infty} \frac{1}{p^{k s}}=\frac{1}{1-\frac{1}{p^{s}}}$. Also, $\frac{-\zeta^{\prime}(s)}{\zeta(s)}$ is sent to $\frac{-\zeta_{p}^{\prime}(s)}{\zeta_{p}(s)}=\frac{\log p}{p^{s}-1}$.

\section{The CASE of the Riemann Zeta Function}

In order to present the main idea behind the proof of Theorem 1 in terms as simple as possible, in this section we show that the Riemann zeta function $\zeta(s)$ and its derivative $\zeta^{\prime}(s)$ are algebraically independent over $\mathbb{C}$. In doing this, we will avoid the use of any analytic properties of the Riemann zeta function, so that we later have a chance of generalizing this reasoning in the context of Theorem 1. where one does not have any assumptions on the convergence of the Dirichlet series associated to $f$, or its Euler product. Returning to the Riemann zeta function, let us assume that $\zeta(s)$ and $\zeta^{\prime}(s)$ are algebraically dependent, and let $Q(x, y)$ be a nonzero polynomial in two variables $x$ and $y$ with coefficients in $\mathbb{C}$ such that $Q\left(\zeta(s), \zeta^{\prime}(s)\right)=0$. Let $P(x, y)=Q(x, x y)$. Then $P(x, y)$ is a nonzero polynomial and $P\left(\zeta(s), \frac{-\zeta^{\prime}(s)}{\zeta(s)}\right)=0$. Next, this gives us an equality in $A_{1}$, namely

$$
P\left(I,-D_{\psi_{l g(I)}} * I^{-1}\right)=0,
$$

where $I \in A_{1}$ denotes the arithmetical function given by $I(n)=1$, and $\psi_{0}$ is the completely additive function given by $\psi_{0}(n)=\log (n)$ for all $n \in \mathbb{N}$. Now for any prime $p$, we apply the homomorphism $\phi_{p, 1,1}$ to the equality (3.1) and find that $P\left(I_{p},-D_{\psi_{0}\left(I_{p}\right)} * I_{p}^{-1}\right)=0$. This in turn gives us an equality between the corresponding Dirichlet series, namely

$$
P\left(\zeta_{p}(s), \frac{-\zeta_{p}^{\prime}(s)}{\zeta_{p}(s)}\right)=0 .
$$


This is a nontrivial relation which needs to be satisfied by each Euler factor $\zeta_{p}(s)$ of $\zeta(s)$ with the same polynomial $P$. On the other hand, one checks by a direct computation that

$$
\frac{-\zeta_{p}^{\prime}(s)}{\zeta_{p}(s)}=\left(1+\zeta_{p}(s)\right) \log p .
$$

Using equation (3.3) in (3.2), we derive that $\zeta_{p}(s)$ is a zero of the polynomial $U_{p}(t)$ which is given by $U_{p}(t)=P(t,(t+1) \log p)$. Since $\zeta_{p}(s)$ is transcendental over $\mathbb{C}, U_{p}(t)$ has to be identically zero. But, since $P(x, y)$ is a nonzero polynomial, $P(t,(t+1) \log p)$ can be identically zero only for finitely many values of $p$, and this completes the proof that $\zeta(s)$ and $\zeta^{\prime}(s)$ are algebraically independent over $\mathbb{C}$.

\section{Proof of Theorem 1}

Let $\psi$ and $f$ be as in the statement of Theorem 1 By our assumptions, we know that there is an infinite set $\mathcal{P}$ of prime numbers with the following property. For each prime $p \in \mathcal{P}$, there exists a component $k_{p} \in\{1, \ldots, r\}$ such that the Dirichlet series associated to the arithmetical function $f_{p, k, r}$ is given by

$$
\bar{f}_{p, k, r}(s)=\bar{f}_{p, k}(s)=\sum_{n=1}^{\infty} \frac{f_{p, k, r}(n)}{n^{s}}=\frac{1}{\left(1-\frac{a_{1}}{p^{s}}\right) \cdots\left(1-\frac{a_{m}}{p^{s}}\right)},
$$

for some $m \in \mathbb{N}$ and nonzero complex numbers $a_{1}, \ldots, a_{m}$. Therefore, there exists a component $k \in\{1, \ldots, r\}$ and an infinite subset $\mathcal{P}_{k} \subseteq \mathcal{P}$ of prime numbers $p$ such that the corresponding values $k_{p}$ are the same and equal $k$.

Fix such an integer $k$ and a prime number $p$ in the subset $\mathcal{P}_{k}$. Let $F(t)$ be defined by $F(t)=F_{p, k, r}(t)=\frac{1}{\left(1-a_{1} t\right) \cdots\left(1-a_{m} t\right)}$. Then, we see that $\bar{f}_{p, k, r}(s)=F\left(p^{-s}\right)$. Let $\psi_{k} \in A_{1}$ be the function defined by $\psi_{k}(n)=\psi(1, \ldots, 1, n, 1, \ldots, 1)$ for all $n \geq 1$, where $n$ occurs at the $k$-th component of the tuple $(1, \ldots, 1, n, 1, \ldots, 1)$ on the right side.

Let $\mathbb{C}(t)$ denote, as usual, the field of rational functions in $t$ over $\mathbb{C}$, and $R^{\prime}(t)$ the derivative of $R(t) \in \mathbb{C}(t)$ as a rational function. Define $\Gamma: \mathbb{C}(t) \rightarrow \mathbb{C}(t)$ by $\Gamma(R(t))=\psi_{k}(p) t R^{\prime}(t)$, for $R(t) \in \mathbb{C}(t)$.

Also define

$$
\bar{f}_{p, k, r}^{\prime}(s)=\bar{f}_{p, k}^{\prime}(s)=\left(\Gamma\left(F_{p, k, r}(t)\right)\right)\left(p^{-s}\right),
$$

and inductively $\bar{f}_{p, k, r}^{(l)}(s)=\bar{f}_{p, k}^{(l)}(s)=\left(\Gamma^{(l)}\left(F_{p, k, r}(t)\right)\right)\left(p^{-s}\right)$ for any positive integer $l$, where $\Gamma^{(l)}$ denotes the composition of $\Gamma$ with itself $l$ times.

Now let $G(t)=G_{p, k, r}(t)=\frac{1}{F_{p, k, r}(t)}$. Then, we find that $G_{p, k, r}(t)$ is a polynomial $G_{p, k, r}(t)=\alpha_{p} t^{m}+\cdots$ with leading coefficient $\alpha_{p}=(-1)^{m} a_{1} \cdots a_{m}$, and its derivative is given by $G_{p, k, r}^{\prime}(t)=m \alpha_{p} t^{m-1}+\cdots$.

Next, define inductively $B_{0}=B_{(p, k, r), 0}=1$ and

$$
B_{n+1}(t)=B_{(p, k, r), n+1}(t)=t\left(G_{p, k, r}(t) B_{n}^{\prime}(t)-(n+1) G_{p, k, r}^{\prime}(t) B_{n}(t)\right) .
$$

We claim that

$$
\begin{aligned}
\Gamma^{n}\left(F_{p, k, r}(t)\right) & =\frac{B_{n}(t)}{(G(t))^{n+1}} \\
& =\frac{B_{n}(t)}{\left(1-a_{1} t\right)^{n+1} \cdots\left(1-a_{m} t\right)^{n+1}} .
\end{aligned}
$$


To prove this claim, first notice that $\Gamma^{0}\left(F_{p, k, r}(t)\right)=F_{p, k, r}=\frac{B_{0}(t)}{G(t)}$ since $B_{0}=1$. Next, assume that $n \geq 1$ and $\Gamma^{n}\left(F_{p, k, r}(t)\right)=\frac{B_{n}(t)}{(G(t))^{n+1}}$. Then,

$$
\begin{aligned}
\Gamma^{n+1}\left(F_{p, k, r}(t)\right) & =\Gamma\left(\Gamma^{n}\left(F_{p, k, r}(t)\right)\right) \\
& =\Gamma\left(\frac{B_{n}(t)}{(G(t))^{n+1}}\right) \\
& =t \frac{b_{n}^{\prime}(t) G(t)^{n+1}-(n+1) B_{n}(t) G(t)^{n} u^{\prime}(t)}{G(t)^{2 n+2}} \\
& =t \frac{b_{n}^{\prime}(t) G(t)-(n+1) B_{n}(t) u^{\prime}(t)}{G(t)^{n+2}} .
\end{aligned}
$$

This completes the proof of the claim.

Observe that $\operatorname{deg}\left(G_{p, k, r}(t)\right)=m$. Now we show inductively that $B_{n}(t)$ is a polynomial of degree $\operatorname{deg}\left(B_{n}(t)\right)=n m$ with leading coefficient $(-1)^{n} \alpha_{p}^{n} m^{n} \psi_{k}(p)$ for all $n \geq 1$. Clearly, $B_{0}(t)$ satisfies this claim. Assume that $n \geq 1$, and $B_{n}(t)$ satisfies the claim. We would like to prove that $B_{n+1}(t)$ satisfies the claim as well; i.e., $B_{n+1}(t)$ is a polynomial of degree $\operatorname{deg}\left(B_{n+1}(t)\right)=(n+1) m$ with leading coefficient $(-1)^{n+1} \alpha_{p}^{n+1} m^{n+1} \psi_{k}(p)$. Since

$$
B_{n+1}(t)=B_{(p, k, r), n+1}(t)=t\left(G_{p, k, r}(t) B_{n}^{\prime}(t)-(n+1) G_{p, k, r}^{\prime}(t) B_{n}(t)\right),
$$

its leading term can be written in the form

$$
\begin{aligned}
& t\left(\alpha_{p} t^{m}\right) n m(-1)^{n} \alpha_{p}^{n} m^{n} \psi_{k}(p) t^{n m-1}-t\left(\alpha_{p}(n+1) m t^{m-1}\right)(-1)^{n} \alpha_{p}^{n} m^{n} \psi_{k}(p) t^{n m} \\
& =\alpha_{p} n m(-1)^{n} \alpha_{p}^{n} m^{n} \psi_{k}(p) t^{(n+1) m}-\alpha_{p}(n+1) m(-1)^{n} \alpha_{p}^{n} m^{n} \psi_{k}(p) t^{(n+1) m} \\
& =(-1)^{n} \alpha_{p}^{n+1} m^{n} \psi_{k}(p)(n m-m(n+1)) t^{(n+1) m} \\
& =(-1)^{n+1} \alpha_{p}^{n+1} m^{n+1} \psi_{k}(p) t^{(n+1) m} .
\end{aligned}
$$

Hence the desired claim holds.

Now let $i, j$ be nonnegative integers such that $i \neq j$. We have that $\bar{f}_{p, k}^{(i)}(s)=$ $\left(\Gamma^{(i)}(F(t))\right)\left(p^{-s}\right)$ and $\bar{f}_{p, k}^{(j)}(s)=\left(\Gamma^{(j)}(F(t))\right)\left(p^{-s}\right)$. Let $S$ denote a finite set of pairs $(u, v)$ of positive integers. Let $P(X, Y) \in \mathbb{C}[X, Y]$ and $P(X, Y)=$ $\sum_{(u, v) \in S} C_{u v} X^{u} Y^{v}$, where $C_{u v}$ is a nonzero complex number for every $(u, v) \in S$.

Suppose that

$$
P\left(D_{\psi}^{i}(f), D_{\psi}^{j}(f)\right)=0 .
$$

By applying the homomorphism $\phi_{p, k, r}$ to both sides of equality (4.1), we find that $P\left(D_{\psi}^{i}(f)_{p, k, r}, D_{\psi}^{j}(f)_{p, k, r}\right)=0$. This in turn gives us an equality between the corresponding Dirichlet series, namely,

$$
P\left(\bar{f}_{p, k}^{i}(s), \bar{f}_{p, k}^{j}(s)\right)=0 .
$$

Thus,

$$
\sum_{(u, v) \in S} C_{u v}\left(\frac{B_{i}(t)}{(G(t))^{i+1}}\right)^{u}\left(\frac{B_{j}(t)}{(G(t))^{j+1}}\right)^{v}=0 .
$$

Let $N=\max _{(u, v) \in S}\{(i+1) u+(j+1) v\}$. We have that

$$
\sum_{(u, v) \in S} C_{u v} B_{i}(t)^{u} B_{j}(t)^{v}(G(t))^{N-(i+1) u-(j+1) v}=0
$$


Note that

$$
\begin{aligned}
\operatorname{deg}\left(B_{i}(t)^{u} B_{j}(t)^{v}(G(t))^{N-(i+1) u-(j+1) v}\right) & =i u m+v j m+(N-(i+1) u-(j+1) v) m \\
& =m(N-u-v) .
\end{aligned}
$$

Let $L=\min _{(u, v) \in S}\{u+v\}$. Then equality (4.3) can be written as

$$
\begin{aligned}
\sum_{\substack{(u, v) \in S \\
u+v=L}} C_{u v} B_{i}(t)^{u} B_{j}(t)^{v}(G(t))^{N-(i+1) u-(j+1) v} & \\
& +\sum_{\substack{(u, v) \in S \\
u+v>L}} C_{u v} B_{i}(t)^{u} B_{j}(t)^{v}(G(t))^{N-(i+1) u-(j+1) v}=0 .
\end{aligned}
$$

For $f \in A_{1}$, consider the support of $f$ given by $\operatorname{supp}(f)=\{n \in \mathbb{N} \mid f(n) \neq 0\}$. By abuse of notation, let us denote by $B_{i}, B_{j}$, and $G$ the arithmetical functions whose Dirichlet series are given respectively by $B_{i}\left(p^{-s}\right), B_{j}\left(p^{-s}\right)$, and $G\left(p^{-s}\right)$. Note that the support of the arithmetical function $\left(B_{i}^{u} B_{j}^{v} G^{N-(i+1) u-(j+1) v}\right)$ is a subset of $\left\{1, p, p^{2}, \ldots, p^{m(N-L)}\right\}$. So the arithmetical function corresponding to the second sum in the above equation, that is, the function given by the sum

$$
\sum_{\substack{(u, v) \in S \\ u+v>L}} C_{u v} B_{i}^{u} B_{j}^{v} G^{N-(i+1) u-(j+1) v},
$$

vanishes at $p^{m(N-L)}$. Since this must hold for infinitely many primes, we conclude that the second sum in the equation above vanishes, and thus

$$
\sum_{\substack{(u, v) \in S \\ u+v=L}} C_{u v} B_{i}(t)^{u} B_{j}(t)^{v}(G(t))^{N-(i+1) u-(j+1) v}=0 .
$$

In this equation, the coefficient of $t^{m(N-L)}$ is

$$
\sum_{\substack{(u, v) \in S \\ u+v=L}} C_{u v}\left(\left(-\psi_{k}(p)\right)^{i} \alpha_{p}^{i} m^{i}\right)^{u}\left(\left(-\psi_{k}(p)\right)^{j} \alpha_{p}^{j} m^{j}\right)^{v}\left(\alpha_{p}\right)^{N-(i+1) u-(j+1) v},
$$

which equals

$$
\sum_{\substack{(u, v) \in S \\ u+v=L}} C_{u v}\left(-\psi_{k}(p)\right)^{i u+j v} \alpha_{p}^{N-u-v} m^{i u+j v} .
$$

We rewrite this sum as

$$
\sum_{\substack{(u, v) \in S \\ u+v=L}} C_{u v}\left(-m \psi_{k}(p)\right)^{i u+j v} \alpha_{p}^{N-L} .
$$

Since the coefficient of $t^{m(N-L)}$ must equal zero, we have that

$$
\sum_{\substack{(u, v) \in S \\ u+v=L}} C_{u v}\left(-m \psi_{k}(p)\right)^{i u+j v} \alpha_{p}^{N-L}=0 .
$$

But, $\alpha_{p} \neq 0$, and so we must have

$$
\sum_{\substack{(u, v) \in S \\ u+v=L}} C_{u v}\left(-m \psi_{k}(p)\right)^{i u+j v}=0 .
$$


By our assumption on $\psi$ and our observation on the set $\mathcal{P}_{k}$ of prime numbers at the beginning of this section, it follows that there exist infinitely many distinct values $\psi_{k}(p)$ for primes in $\mathcal{P}_{k}$. Each of these values $\psi_{k}(p)$ must satisfy (4.4), which is not possible. This completes the proof of Theorem 1 .

Proof of Corollary 1, Let $f$ and $\psi$ be as in the statement of Corollary 1, Let $Q \in \mathbb{C}[f]$ be such that $D_{\psi}(Q) \in \mathbb{C}[f]$. Since for any $c \in \mathbb{C}$ and $n \in \mathbb{N}, D_{\psi}\left(c f^{n}\right)=$ cn $f^{n-1} D_{\psi}(f), D_{\psi}(Q)$ equals $D_{\psi}(f)$ times a polynomial in $f$. But, $f$ and $D_{\psi}(f)$ being algebraically independent, the only multiple of $D_{\psi}(f)$ inside $\mathbb{C}\left[f, D_{\psi}(f)\right]$ which belongs to $\mathbb{C}[f]$ is zero, and this proves the corollary.

\section{REFERENCES}

[1] E. Alkan, A. Zaharescu, M. Zaki, Arithmetical functions in several variables, Int. J. Number Theory 1 (2005), no. 3, 383-399. MR2175098 (2006k:11008)

[2] E. Alkan, A. Zaharescu, M. Zaki, Multidimentional averages and Dirichlet convolution, Manuscripta Math. 123 (2007), 251-267. MR2314084 (2008d:11089)

[3] B. Berndt, K. Ono, Ramanujan's unpublished manuscript on the partition and tau functions with proofs and commentary, Sém. Lothar. Combin. 42 (1999). MR1701582 (2000i:01027)

[4] E.D. Cashwell, C.J. Everett, The ring of number-theoretic functions, Pacific J. Math. 9 (1959), 975-985. MR0108510 (21:7226)

[5] W. Narkiewicz, On a class of arithmetical convolutions, Colloq. Math. 10 (1963), 81-94. MR0159778 (28:2994)

[6] W. Narkiewicz, Some unsolved problems, Colloque de Théorie des Nombres (Univ. Bordeaux, Bordeaux, 1969), pp. 159-164. Bull. Soc. Math. France, Mem. No. 25, Soc. Math. France, Paris, 1971. MR0466060 (57:5943)

[7] K. Ono, The Web of Modularity: Arithmetic of the Coefficients of Modular Forms and $q$-series, CBMS Regional Conference Series in Mathematics 102. Published for the Conference Board of the Mathematical Sciences, Washington, DC, by the American Mathematical Society, Providence, RI, 2004. MR2020489 (2005c:11053)

[8] S. Ramanujan, Collected Papers, Chelsea, New York, 1962.

[9] A. Schinzel, A property of the unitary convolution, Colloq. Math. 78 (1998), no. 1, 93-96. MR.1658143 (99k:11010)

[10] E. D. Schwab, Möbius categories as reduced standard division categories of combinatorial inverse monoids, Semigroup Forum 69 (2004), no. 1, 30-40. MR2063975 (2005b:20121a)

[11] E. D. Schwab, The Möbius category of some combinatorial inverse semigroups, Semigroup Forum 69 (2004), no. 1, 41-50. MR2063976 (2005b:20121b)

[12] E. D. Schwab, G. Silberberg, A note on some discrete valuation rings of arithmetical functions, Arch. Math. (Brno) 36 (2000), 103-109. MR1761615 (2001d:13022)

[13] E. D. Schwab, G. Silberberg, The valuated ring of the arithmetical functions as a power series ring, Arch. Math. (Brno) 37 (2001), 77-80. MR1822767

Department of Mathematics, University of Illinois at Urbana-Champaign, 1409

W. Green Street, Urbana, Illinois 61801

E-mail address: zaharesc@math.uiuc.edu

Department of Mathematics, University of Illinois at Urbana-Champaign, 1409

W. Green Street, Urbana, Illinois 61801

E-mail address: mzaki@math.uiuc.edu 